\title{
Participación política de los jóvenes del Valle del Cauca, Colombia, en la toma de decisiones públicas
}

\author{
Luis Eduardo Gutiérrez-Rojas \\ Politólogo \\ Universidad del Valle del Cauca. Cali, Colombia \\ https:/ / orcid.org/0000-0002-9688-2399 • luis.g.rojas@correounivalle.edu.co
}

\section{Amanda Ramírez-Giraldo}

Magíster en políticas públicas. Administradora de empresas

Universidad del Valle del Cauca. Cali, Colombia

https:/ / orcid.org/0000-0001-5096-6055• amanda.ramirez@correounivalle.edu.co

Resumen

Este artículo expone los principales resultados de la investigación Participación política de los jóvenes del Valle del Cauca en la toma de decisiones de política pública, derivado de la investigación que lleva el mismo título que se adelantó en el marco de la maestría en políticas públicas de la Universidad del Valle. El objetivo de esta investigación fue identificar la capacidad de incidencia que tienen los jóvenes en la toma de decisiones en política pública en el Valle del Cauca, entre los años 2005-2015, empleando un análisis de caso múltiple (4) a través de dos herramientas metodológicas: la entrevista a profundidad y los relatos de vida. La investigación arrojó, como principal conclusión, que los jóvenes logran movilizar sus demandas hacia el Estado influenciando en todo el ciclo de la gestión pública, especialmente en la elaboración del diagnóstico y en la formulación. Sin embargo, su capacidad de incidencia política está estrechamente relacionada con la cercanía que tengan a los tomadores de decisión: entre más alejado se esté de los decisores, menor es la influencia y viceversa. Dicha distancia es dada por el tipo de liderazgo ejercido por los jóvenes que fueron objeto de estudio en esta investigación, cada tipología determina al mismo tiempo la distancia.

Palabras clave: Participación política; Participación juvenil; Toma de decisiones; Elaboración de políticas públicas.

Recibido: 15/01/2021 |Aprobado: 16/04/2021 |Publicado: 01/07/2021

(c) (1)@(2) Esta obra está bajo una Licencia Creative Commons Atribución-NoComercialCompartirIgual 4.0 Internacional.

Financiación o proveniencia del artículo: Artículo derivado de la investigación Participación política de los jóvenes del Valle del Cauca en la toma de decisiones de política pública 2005-2015 en el marco de la Maestría en Políticas Públicas de la Universidad del Valle.

¿Cómo citar este artículo? / How to quote this article?

Gutiérrez-Rojas, L. E., y Ramírez-Giraldo, A. (2021). Participación política de los jóvenes del Valle del Cauca, Colombia, en la toma de decisiones públicas. Prospectiva. Revista de Trabajo Social e intervención social, (32), 103-124. doi: 10.25100/prts.v0i32.10123. 
Gutiérrez-Rojas y Ramírez-Giraldo

\title{
Youth Political Participation in Policy Decision Making in Valle del Cauca, Colombia
}

\begin{abstract}
This article is a product of a research study named Youth political participation in policy decision making in Valle del Cauca conducted as part of the Public Policy master's degree program in Universidad del Valle. The main objective was to identify the incidence that young people had in the public policy decision making process in Valle del Cauca, Colombia, between 2005 and 2015. The study employed multiple case study (4) analysis through two methodological tools: in-depth interviews and narration of life experiences. The main conclusion was that young people are able to mobilize their demands to the State by influencing the entire cycle of public policy management, and especially, in agenda setting, problem definition and policy formulation. However, their incidence level is deeply related with their closeness to decision makers, and such closeness is determined by the type of leadership exercised by the young people.
\end{abstract}

Key words: Political participation; Youth participation; Decision making; Policy making.

Sumario: 1. Introducción, 2. Metodología, 3. Hallazgos, 4. Conclusiones, 5. Referencias bibliográficas. 


\section{Introducción}

La participación política ha sido uno de los temas centrales de estudio de las ciencias sociales ya que es un fenómeno que permite comprender los sistemas políticos occidentales democráticos, cuyas acciones van desde el hecho del voto hasta la participación activa en procesos de toma de decisión en política pública o ejerciendo control social sobre la actividad de los gobiernos.

La participación política se refiere a una serie de actividades y comportamientos realizados por la ciudadanía de una comunidad política que los ubican en un entorno relacional con diferentes instituciones públicas y privadas. Ya sean solamente como receptores de información política, como dirigentes y coordinadores de mítines o incluso desafiando al orden establecido, todas las personas están, de uno u otro modo, participando de la vida política (Bobbio y Mateucci, 1981).

A partir de estudios cualitativos realizados en el orden global, Chile (Loreto-Martínez, Silva, Morandé y Canales, 2010), México (López Serrano y López Serrano, 2009) y Colombia Botero-Gómez, Torres-Hincapié y Alvarado, 2008), este fenómeno ha entrado en la agenda pública como un tema que ha cautivado la atención de académicos y líderes políticos. Estos debates han girado sobre diversos aspectos: la débil participación juvenil en los asuntos políticos, en las elecciones y en los espacios de debate (Acosta-Valencia y Garcés-Montoya, 2012; Parker-Gumucio, 2003); la marginación que la población juvenil sufre debido a prejuicios y prenociones de quienes ejercen el poder (González-Sancho y HenríquezCáceres, 2017; Hopenhayn, 2004); la importancia del concepto de ciudadanía juvenil que ha sido implementado en la legislación nacional (Loreto-Martínez, Silva, Morandé y Canales, 2010; Benedicto, 2016; Botero-Gómez, Torres-Hincapié y Alvarado, 2008); el papel que han desempeñado las redes sociales, tanto en el activismo juvenil, como en la política electoral (Niño-Lim; 2009; Fisher, 2012); hasta formas de expresión y comunicación alternativas manifestadas a través de actividades artísticas, agrícolas, sociales, ambientales o comunitarias (Hurtado-Galeano, 2010).

Colombia se ha caracterizado por ser un país con bajos niveles de participación, con tasas de abstención electoral elevadas y con un marcado ausentismo en las urnas electorales (Díaz-Hernández y Moreno-Martínez, 2017). Las encuestas y diferentes estudios muestran que la cultura política del país tiende a ser, bajo la clasificación de Almond y Verba (2001), una cultura súbdita, que es consciente de la existencia de autoridades públicas, pero que no genera una relación de retroalimentación con dichas instituciones.

Los jóvenes no escapan a esta realidad, pues sus niveles de participación electoral tienden a ser más bajos que la media nacional, tal como lo demuestran Díaz-Hernández y Moreno-Martínez (2017). Adicionalmente, las estadísticas muestran que la participación 
Gutiérrez-Rojas y Ramírez-Giraldo

política no electoral es baja en el país: la encuesta de cultura política que realiza el Departamento Nacional de Estadística [DANE], permite plasmar, empíricamente, las afirmaciones que se han hecho. Los porcentajes de participación en organizaciones sociales son bajos; no hay incentivos para organizarse y buscar acción política colectiva; no se conocen los mecanismos o espacios de participación política; hay una desconfianza hacia las instituciones políticas y hacia la práctica política (Departamento Nacional de Estadística [DANE], 2019).

Ante este panorama de baja participación política juvenil, surge la inquietud sobre el escenario regional: conocer sobre la participación de los jóvenes en los procesos electorales territoriales, evidenciar su comportamiento en los espacios institucionales creados por el Estado para la interlocución con la sociedad civil y caracterizarlos, y describir y analizar la oferta institucional que tiene el Estado en materia de participación ciudadana.

El presente artículo indaga sobre las experiencias de participación política juvenil que han tenido diversos jóvenes en el departamento del Valle del Cauca para dar respuesta al principal interrogante que guio la investigación: ¿cómo incide la participación política de los jóvenes en los procesos de toma de decisión en política pública en la escala regional? Para dar respuesta a esta cuestión, la investigación se planteó un objetivo central: identificar la capacidad de incidencia que tienen los jóvenes en la toma de decisiones en política pública en el Valle del Cauca, entre los años 2005-2015.

\section{Metodología}

El enfoque metodológico utilizado es cualitativo y se cimienta en dos grandes campos disciplinares: la ciencia política y las políticas públicas. La primera, permitió la elaboración de los componentes teóricos relacionados con democracia y el concepto de participación; la segunda, permitió, a través de la mirada de policy cycle, enmarcar la participación en relación con los procesos de toma de decisión de política pública en cualquier ciclo de esta, ya sea formulación, implementación o evaluación.

La investigación es un estudio de caso múltiple ${ }^{1}$ en donde se reconstruyeron, a partir del empleo de la entrevista de investigación ${ }^{2}$, cuatro relatos de vida de jóvenes que han participado en procesos de toma de decisión en política pública. Los relatos permiten el análisis de acontecimientos, vivencias y sucesos de vida de una persona e indaga sobre los

1 De acuerdo con Yin (2017) los estudios de caso múltiple se usan para explorar diferencias entre casos y dentro de ellos mismos con el objetivo de replicar los hallazgos.

2 La entrevista se define como una conversación que se propone un fin distinto al hecho de conversar, pues adquiere un aspecto técnico con el objetivo de recabar información (Díaz-Bravo, Torruco-García, MartínezHernández y Varela-Ruiz, 2013, p. 163). Las entrevistas semiestructuradas tienen un alto grado de flexibilidad porque parten de preguntas preestablecidas, pero las cuales pueden ajustarse a medida que la entrevista avanza. 
Gutiérrez-Rojas y Ramírez-Giraldo

sentimientos, las maneras de entender y comprender el mundo por parte del sujeto investigado. Con los relatos de vida se dibuja el perfil cotidiano de una persona mediante el empleo de la entrevista o incluso a través de conversaciones sostenidas entre investigador e investigado.

Para la selección de los cuatro casos de estudio se hizo una preselección entre 16 jóvenes que se seleccionaron a partir de dos factores: el marco teórico y su experiencia en procesos de participación política. Respecto a lo primero, la investigación realizó una definición de la categoría de participación política a partir de la obra de Cordourier-Real (2015) y Pasquino (2011). Este último define la participación política como el "conjunto de acciones y de conductas que apuntan a influir de manera más o menos directa y más o menos legal sobre las decisiones, así como la misma selección de los detentadores del poder en el sistema político o en cada organización política" (p. 70). A partir de esta definición, Cordourier-Real (2015) elabora una tipología de la participación política que establece dos grandes categorías de participación que se dan a partir de dos sistemas de libertades que abarcan un conjunto de derechos: el sistema de libertades político-electorales y el sistema de libertades civiles. El primero se refiere a un conjunto de derechos que permite la competencia en el ámbito electoral, en otras palabras, las elecciones y todo lo que implica (formación de cuadros al interior de los partidos, elecciones primarias, encuestas de opinión, etc.). El segundo se refiere a derechos no vinculados con procesos electorales, pero permite la comunicación de ideas políticas y la asociación política para tramitar demandas, participación en marchas, paros o protestas, etc.

Tomando como base estos dos sistemas, el autor define tres tipos de participación: la participación electoral (participación convencional), relacionada con procesos como el sufragio universal; la política no electoral, regularmente antagónica y que se subdivide en participación alternativa y participación cívica, la primera, relacionada con formas de participar alternativas no ligadas a canales institucionales (mítines, protestas, redes sociales, denuncia ciudadana); la segunda, que se refiere a la pertenencia a grupos y organizaciones de participación voluntaria (ambas, formas de participación no convencionales). Esta última, de acuerdo con el autor, no busca siempre construir demandas que sean procesadas por el sistema político, sino que tiene un componente simbólico. A estas tres categorías, la investigación añadió una cuarta que surgió como categoría emergente luego de un proceso de análisis normativo y de las entrevistas: la participación institucional, la cual se da en los canales que el poder ejecutivo, tanto a nivel nacional como territorial, crea para dialogar con la ciudadanía. La Figura 1 muestra, gráficamente, esta clasificación de dos categorías. 
Gutiérrez-Rojas y Ramírez-Giraldo

Figura 1. Categorías de participación ciudadana.

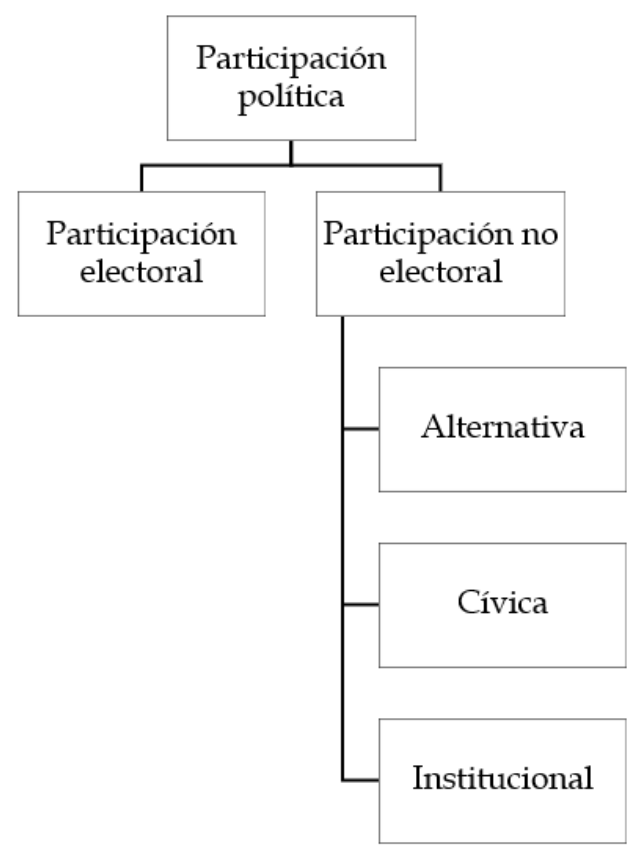

Fuente: elaboración propia a partir de Cordourier-Real (2015).

En el campo de las políticas públicas hay que partir de dos autores que dan el fundamento: Müller (2006), con las nociones del enfoque cognitivo y la importancia de las ideas en el campo de la toma de decisiones, y Roth-Deubel (2017), que define, de manera clara, lo que es una política pública: una intervención del Estado, enmarcada dentro de un documento jurídico, que traza un plan de general de acciones que se concretan a través de planes, programas y proyectos.

Esta definición, dirá Roth-Deubel, es acertada, aunque limitada porque tiene un sesgo claramente estatocentrista y en los estados contemporáneos hay una multiplicidad de actores políticos, comunitarios, económicos o sociales que participan en la toma de decisiones. Además de eso, esta mirada centrada en el Estado lo concibe como una organización reactiva, es decir, como una institución que reacciona ante un estímulo y juega un rol pasivo en el escenario social.

En lo que respecta al ciclo de la política, este enfoque permite descomponer la política en cinco fases: 1. Identificación del problema: la situación problema es percibida por actores políticos y sociales representados por partidos, gremios y sindicatos. Se busca que el problema se inscriba en la agenda de gobierno (agenda setting); 2. Formulación de soluciones: la administración propone una o varias soluciones (policy formulation); 3 . Toma de decisiones: se examinan las soluciones y se deciden las acciones y decisiones; 4. 
Gutiérrez-Rojas y Ramírez-Giraldo

Implementación: por lo general, es la administración la encargada de implementar o no la solución; 5. Evaluación: los actores evalúan (policy evaluation) y se reajusta la política (feedback) o se suprime (Kelly, 2003). Retomar el ciclo de la política es clave para esta investigación porque en las primeras etapas se encuentran dos momentos donde la participación política juega un papel fundamental: la identificación del problema y la inscripción en la agenda.

Una vez definido el factor teórico con la construcción de las cuatro categorías ideales de participación, se preseleccionaron 16 posibles sujetos mediante una ficha de caracterización en donde se condensó el segundo factor: la experiencia de participación. Para la elaboración de estas fichas se realizaron entrevistas cortas, de 30 minutos de duración. Posteriormente, se seleccionaron los cuatro relatos de vida, un relato por cada categoría de participación con una observación fundamental: estas categorías nunca son excluyentes o dicotómicas entre sí, por el contrario, se traslapan; un sujeto puede tener una trayectoria de participación política en donde las cuatro categorías estén presentes. Sin embargo, la investigación dejó entrever que cada sujeto potencial, a pesar de tener experiencia bajo todas las modalidades (electoral y no electoral), siempre tiene una que prima sobre las demás.

El paso siguiente fue la formulación de los criterios de selección de los cuatro casos. Para la presente investigación, el primer criterio fue que cada uno de los sujetos entrevistados hubiese participado políticamente, en algún momento de su trayectoria y bajo cualquier categoría de participación de las expuestas, en procesos de toma de decisión en política pública. El segundo criterio es que dicha participación se haya dado cuando los sujetos eran aún jóvenes bajo los parámetros de la legislación colombiana. Esto es así por dos razones principales: en primer lugar, porque una de las pretensiones del trabajo investigativo era no ser coyuntural, esto permite tener una mirada de sucesos previos que no estén permeados por la inmediatez de la ocurrencia del fenómeno. En segundo lugar, porque la historia de vida analiza procesos continuos y no sucesos específicos limitados a un lapso particular.

Se tomaron en consideración otros aspectos fundamentales que, sin embargo, no fueron criterios de selección claves como sí ocurrió con los dos anteriores: la edad, pues la mayoría de los preseleccionados cumplieron este criterio; el sexo y el género para la selección de los casos; la pertenencia étnica que manifestaron algunos de los sujetos identificados en la ficha; la territorialidad y la cantidad de actividades realizadas por cada sujeto en cada categoría.

A los cuatro seleccionados se les aplicó una entrevista semiestructurada y a profundidad las cuales se relacionaron con los objetivos específicos y con las preguntas derivadas de investigación: 1 . ¿Cómo tramitan los jóvenes sus demandas frente al estado en 
Gutiérrez-Rojas y Ramírez-Giraldo

el nivel territorial?; y 2. ¿De qué manera se movilizan políticamente los jóvenes? A partir de estas dos preguntas guías se elaboraron un total de diez preguntas.

Para el análisis de los resultados se empleó el software Atlas.ti con el objetivo de realizar codificación abierta ${ }^{3}$ relacionando dos aspectos: por un lado, las categorías de participación previamente expuestas (participación electoral, participación alternativa, participación cívica y participación institucional); por el otro, a partir de la construcción del problema en la investigación, se encontró que la participación política se mediaba en dos dimensiones, una interna y una externa. La primera, refiere al componente subjetivo del sujeto (creencias, postura hacia la política, creencias, desconfianza a las instituciones, formación democrática, etc); la segunda, se refiere a componentes objetivos (restricciones legales de participación política electoral ${ }^{4}$, estructuras de poder adultocentrista ${ }^{5}$ ). La Tabla 1 muestra la relación entre las categorías de participación y las dimensiones.

Tabla 1. Co-ocurrencia de variables para el análisis de la entrevistas.

\begin{tabular}{cccc}
\hline Categoría & Subcategoría & $\begin{array}{c}\text { Dimensión interna } \\
\text {-Apatía política } \\
\text {-Educación } \\
\text { democrática }\end{array}$ & $\begin{array}{c}\text { Dimensión externa } \\
\text {-Restricciones legales } \\
\text {-Estructuras de poder } \\
\text { (adultocentrismo) }\end{array}$ \\
\hline \multirow{2}{*}{$\begin{array}{c}\text { Participación } \\
\text { política }\end{array}$} & Electoral & A.1 & B.1 \\
& Alternativa & A.2 & B.2 \\
& Institucional & A.3 & B.3 \\
\hline
\end{tabular}

Fuente: elaboración propia.

Finalmente, se construyeron cuatro relatos que obedecen a los cuatro casos y se graficaron sus trayectorias en líneas de tiempo a partir de la identificación de hitos que surgieron luego del análisis.

3 Se entiende por codificación abierta la evaluación minuciosa de las entrevistas y el proceso analítico mediante el cual se identifican los conceptos, se descubren las propiedades y se encuentran las dimensiones (Strauss y Corbin, 2002).

4 Las restricciones constitucionales establecidas en los artículos 172 y 177 de la Constitución Política que impide a los jóvenes ser congresistas (para ser senador se requiere ser mayor de 30 años y para ser representante, tener más de 25 años). En Colombia, se es legalmente joven entre los 14 y 28 años.

5 El concepto de adultocentrismo fue esencial en la investigación. Este se define como los prejuicios generados por los adultos hacia la figura del joven como actor político y su instrumentalización en los discursos políticos (Acosta-Valencia y Garcés-Montoya, 2012; González-Sancho y Henríquez-Cáceres, 2016; Hopenhayn, 2004) 


\section{Hallazgos}

Como previamente se había especificado, los cuatro casos estudiados responden a una estrategia investigativa: en un primer momento se debe tener claridad de las definiciones ya plasmadas, posteriormente se construyeron unos tipos ideales que van a ser las guías en la selección para luego llegar a un supuesto: los cuatro tipos de participación política nunca se presentan en su forma pura, sin embargo, cada sujeto tiene dentro de su trayectoria de vida un aspecto de participación que prima sobre los demás y a partir de ese criterio fue seleccionado.

Así las cosas, la estructura narrativa detalla los resultados caso por caso siguiendo un modelo secuencial-cronológico: se parte de los inicios en los procesos políticos de cada sujeto, posteriormente se especifican las motivaciones que fueron determinantes para participar, luego se procede a relatar sus trayectorias y experiencias, en cuarto lugar se determina si en su participación política incidió la implementación de políticas públicas para jóvenes y, por último, se redactan las conclusiones a partir del análisis de aspectos comunes.

Cada uno de los casos expuestos en los hallazgos, inicia con una gráfica que expone, a manera de línea de tiempo, los principales hitos de cada trayectoria política. Así, para el caso electoral, alternativo, cívico e institucional, se inicia con dicha figura (Figura 2) que introduce al lector en el relato que se construye a continuación.

\section{Caso 1. Electoral}

Figura 2. Trayectoria, caso electoral.

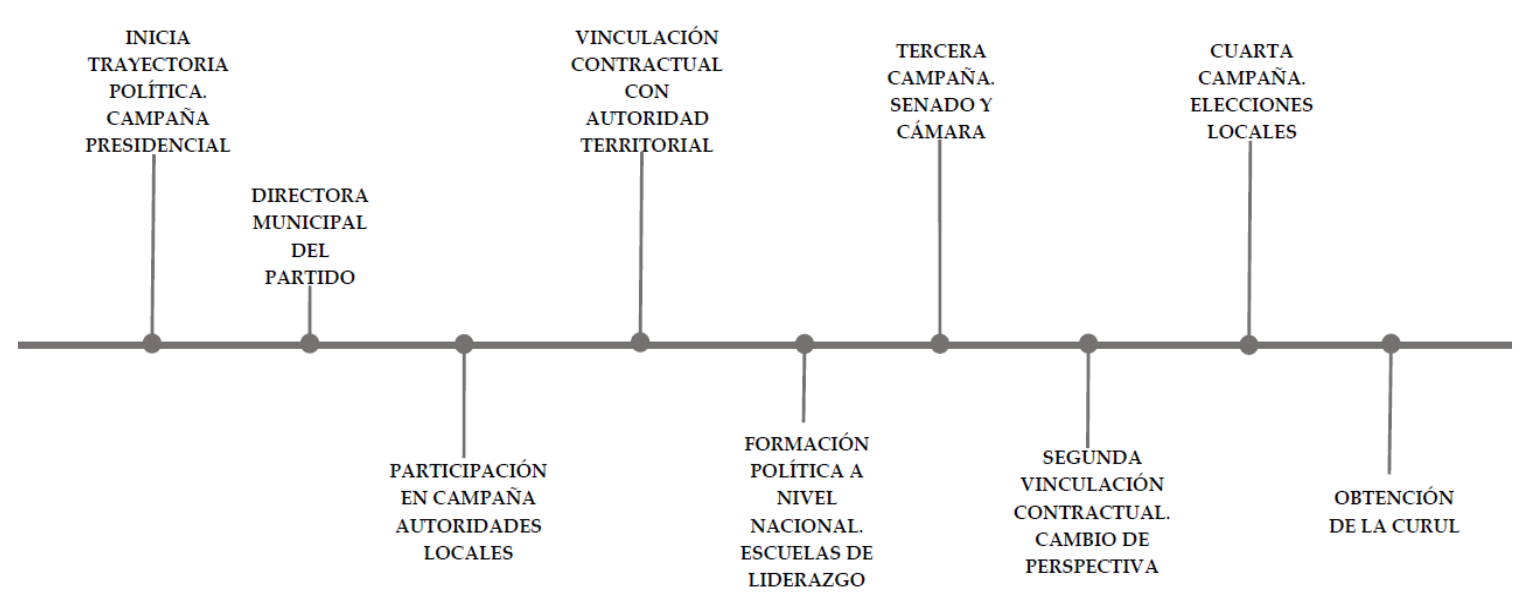

Fuente: elaboración propia. 
Gutiérrez-Rojas y Ramírez-Giraldo

El caso electoral es una mujer que ocupa una curul en una corporación de elección popular a nivel territorial. Inició sus actividades políticas cuando cursaba la educación secundaria, haciéndose elegir personera; posteriormente se ligó con la participación electoral acompañando una campaña presidencial en el año 2010, siendo aún menor de edad, desde los espacios universitarios.

La principal motivación que, de acuerdo con lo manifestado por la entrevistada, la incentivó a participar de una campaña política fue un aspecto de carácter moral ligado a un deber ciudadano "yo quiero hacer campaña, yo quiero hacer política porque son ellos los que toman decisiones y si nosotros los ciudadanos nos seguimos manteniendo alejados, pues las van a tomar mal".

Haber sido tan joven y ser directora municipal de un partido político trajo sus primeros encuentros y experiencias con las estructuras de poder "adultocentrista". En medio de una campaña electoral territorial, consiguió que su partido le otorgara un aval a un candidato a la alcaldía de su municipio, quien en un principio fue escéptico de esta posibilidad debido a la juventud que tenía ella. Dicho candidato sería elegido alcalde de su municipio por el mismo partido y vincularía, contractualmente, a la investigada en la administración municipal.

La entrevistada manifestó que, luego de su paso por la alcaldía, comenzó un proceso de formación política en todo el país con el fin de conformar un equipo de juventudes para el partido a nivel nacional, aunque esta actividad se ligaba directamente con lógicas partidistas, los procesos de formación en los que participó se clasificaron como alternativos, ya que recorrió diferentes universidades públicas colombianas y participó en diversos debates y asambleas estudiantiles en el marco de manifestaciones y paros.

Esta experiencia permitió constatar que las estructuras de poder adultocentrista no son las únicas barreras actitudinales con que la entrevistada se encontró, pues en los espacios de formación universitarios su condición de mujer también le hizo merecedora de críticas y comentarios descalificadores, "a mi cualquier otro me ganaba la discusión, me ganaba el pulso y yo como mujer y además tan joven pues todos me decían: 'es que está muy chiquita, está muy niña'".

Posterior a este proceso de formación política, la senda electoral continuó con elecciones nacionales, apoyando diferentes campañas al Senado de la República y a la Cámara de Representantes. La candidata apoyada a esta última corporación resultó victoriosa. Como la investigada reconoce, en este punto de su trayectoria su percepción de la política tuvo un cambio, "yo comienzo a hacer un proceso más pragmático, más concentrado en buscar votos que en buscar quiénes eran afines ideológicamente al proceso". 
Gutiérrez-Rojas y Ramírez-Giraldo

Una vez su partido político obtuvo la victoria en la Cámara de Representantes, la entrevistada asumió un rol operativo en la organización política al convertirse en coordinadora de las relaciones políticas entre la alcaldía, los concejales y la representante a la cámara, retomando su anterior cargo en la alcaldía municipal:

Me pongo a hacer el enlace de los concejales con el acalde municipal. Ahí termina uno volviéndose aún más pragmático. Ya entiende otras dinámicas de la política, otras dinámicas del poder. Ya uno entra a otra etapa de la vida política donde tiene que aprender, conocerlas, saber cómo funcionan y eso me tocó a mí en esos años de alcaldía: saber cómo era el tema con los concejales y el manejo del concejo municipal, que fue un manejo durísimo y que eran pulsos muy berracos.

Nuevamente, la tensión entre ella como joven y las estructuras de poder adultas se hicieron evidentes, pues se generaron múltiples tensiones con los concejales. La cuestión de género también estaba presente, ya que la investigada, al ser la mano derecha del alcalde, comenzó a coordinar los asuntos de contratación. Es este último aspecto lo que le da la posibilidad de solventar estos conflictos, ya que, de acuerdo con su versión, todos los contratos debían pasar por su revisión antes de la firma del alcalde.

El alcalde no firmaba un solo contrato sin que yo le diera el visto bueno. Me di cuenta de que no necesitaba hablar más fuerte que otro, sino que con un lapicero tenía yo el poder. Entendí las dinámicas de poder y las diferencias de los liderazgos, hay quienes están acostumbrados a hacer liderazgo apabullante, mirando cómo habla del otro, cómo pasa por encima del otro, hay quienes son protagonistas y hay quienes entienden la dinámica del poder que saben que no tienen por qué enemistarse con nadie, construir a partir de las diferencias y a sentarse a hablar.

Su participación electoral también se vio traslapada con otro tipo de participaciones que le permitieron formarse como líder y lograr así una mayor incidencia en la toma de decisiones. Hizo parte de la mesa multipartidista del Departamento, desde donde pudo opinar de diferentes aspectos relacionado con la juventud:

En la primera mesa multipartidista yo me senté al lado del Polo y el Polo se sentó al lado mío porque nos sentíamos afines y podíamos conversar. El partido Conservador se sentó en esa época con el partido de la U. Cuando rompíamos esas barreras nos dábamos cuenta de que al final todos éramos jóvenes y que estábamos ahí sentados para hablar del tema de la juventud. 
Gutiérrez-Rojas y Ramírez-Giraldo

\section{Caso 2. Alternativo}

Figura 3. Trayectoria, caso alternativo.

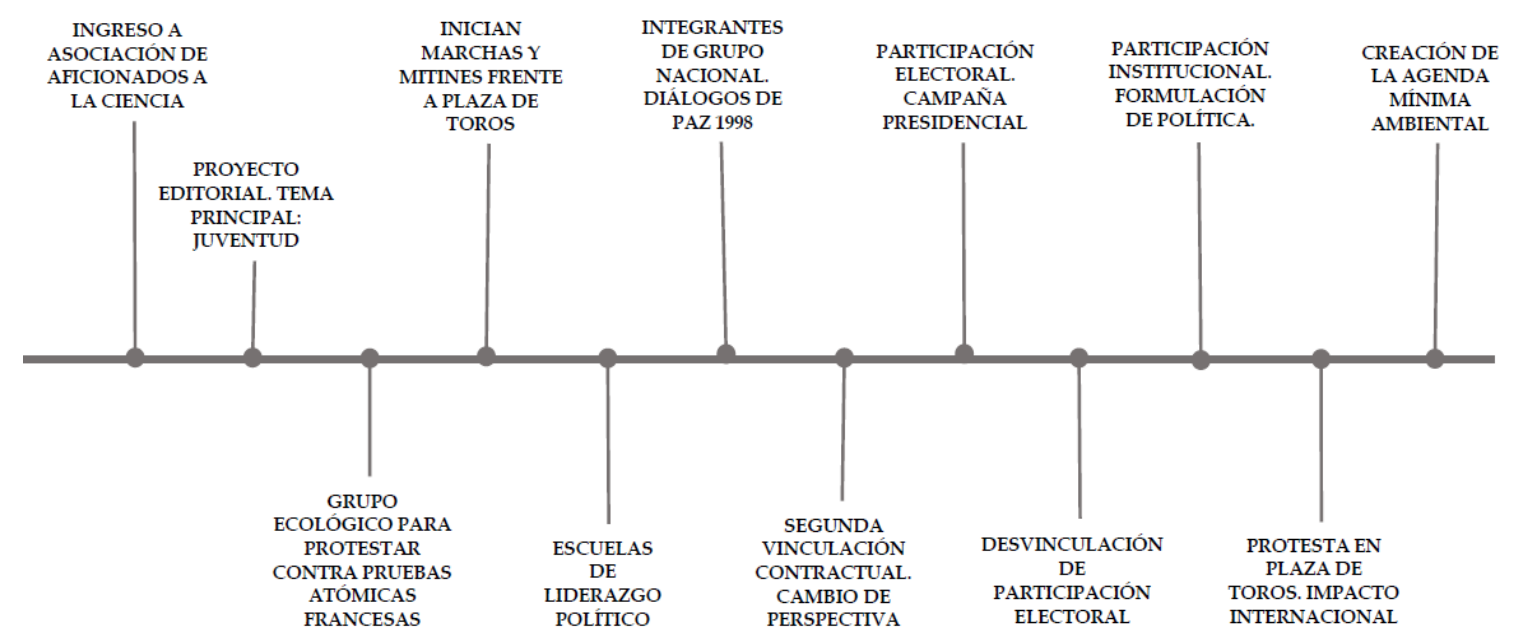

Fuente: elaboración propia.

El segundo caso de estudio (Figura 3) es un hombre que ha sido un activista de dos causas: defensa de los derechos de los animales y el ambientalismo. A partir de diferentes espacios que se relacionan principalmente con estos dos elementos, ha construido un liderazgo político que lo llevó a participar en elecciones para el Congreso de la República. El patrón se repite: a pesar de que su trayectoria orbita en la subcategoría alternativa, hay experiencias que se inscriben en las otras clasificaciones expuestas por Courdourier-Real (2015).

Los inicios de la trayectoria del sujeto investigado no se relacionan con la actividad política propiamente dicha, el entrevistado situó sus primeras experiencias en relación con la actividad científica como un punto importante que generó en él su interés por los temas que lo movilizarían en un futuro.

Se vinculó a organizaciones y colectivos sociales donde el tema principal era el activismo antitoreo el cual cobró especial relevancia en la trayectoria del investigado, pues empezó a participar y a organizar manifestaciones y movilizaciones contra esta práctica, así como diferentes mítines frente a la plaza de toros de la ciudad. Este tema marcaría su liderazgo político hasta la fecha en que se escribe este documento. 
Gutiérrez-Rojas y Ramírez-Giraldo

A partir de estas experiencias, el joven manifestó que se gestó en él un sentimiento antipolítico, el cual surgió porque conoció de diferentes personas que laboraban en el sector público y cuyo empleo dependía de la consecución de votos para la conservación de estos. Sin embargo, también existe en él una presencia del segundo componente de las dimensiones internas, la educación democrática. A pesar de la apatía y el rechazo a las prácticas políticas que ya se mencionaron, hizo parte de diversos procesos formativos en cuestiones políticas, uno fomentado por una organización no gubernamental, otro promovido por un programa de liderazgo juvenil financiado con fondos estatales y un tercero adicional fomentado por los grupos juveniles del partido comunista.

Se vinculó a dos campañas electorales, una a nivel territorial y otra para presidenciales. En esta última fue designado como coordinador de juventudes y empezó a liderar movilizaciones en pro de la campaña, sin embargo, se encontró con las barreras actitudinales adultocentristas:

Yo hice una propuesta de las cosas que pensaba, de cómo se podía hacer una campaña chévere, pero no me pararon bolas, esa campaña tampoco tenía muchos recursos... Yo proponía actividades en la calle, no adentro sino en la calle, pero culturales, proponía foros, conversatorios, actividades académicas, debates... Era un poco hacer lo que venía haciendo, pero ya en este contexto de elecciones y no, eso no tuvo acogida.

A raíz de esta actitud reticente hacia sus propuestas y debido a la inactividad de la campaña, el entrevistado manifestó que la apatía hacia la política se incrementó, alimentada también por el acercamiento a lecturas políticas críticas del Estado desde miradas marxistas y anarquistas. Posteriormente, él decidió continuar con el activismo en diferentes organizaciones sociales y dejar lo electoral de lado. Pero como los tipos de participación que se han definido nunca se presentan en su forma ideal, el sujeto fue también partícipe de la construcción de la política pública de juventud de su ciudad.

El activismo se hizo más fuerte con el tema antitaurino, organizando manifestaciones y mítines frente a la plaza de toros. Una de dichas prácticas cautivó la atención de medios de comunicación internacionales, fue cuando, desde las graderías de la plaza saltó al centro del ruedo y fue expulsado de manera violenta por el personal de seguridad de la institución. Esto logró un posicionamiento del tema en la agenda pública:

Antes el tema era tabú, censurado, particularmente el tema antitoreo, los periodistas se morían de miedo de solo hablar de toreo, hasta de perros y gatos te hablaban, de ahí para allá, te decían: 'corridas no, los dueños del periódico son accionistas... me echan'. 


\section{Caso 3. Cívico}

Figura 4. Trayectoria, caso cívico.

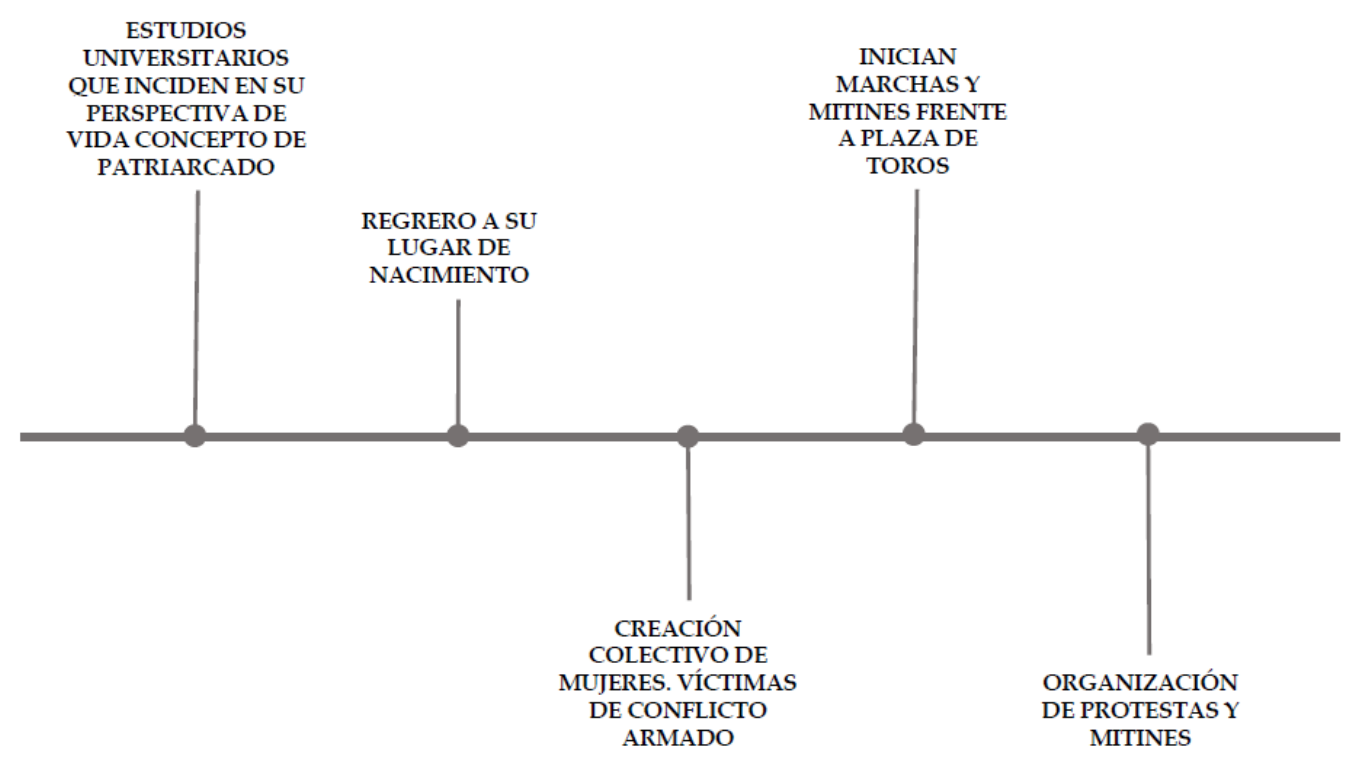

Fuente: elaboración propia.

El tercer caso de estudio (Figura 4) reúne unas características que lo hacen diferente a los otros. Es una mujer, afrodescendiente y habita en la zona rural de un municipio con amplios porcentajes de población afrodescendiente. Estos elementos configuran las experiencias de participación de una manera diferente, pues la ubican en contextos específicos que inciden sobre la manera en que ella ha asumido su rol en la participación política, la cual se ha enfocado, principalmente, hacia los asuntos de género, de mujer y de lo que ella denomina defensa del territorio.

El concepto de patriarcado fue de importancia en su movilización, pues fue uno de los principales impulsos para adelantar actividades organizativas con otras mujeres que habitaban en el territorio.

Nosotras como mujeres no sabíamos qué era el concepto, pero luego de darnos cuenta de qué era el patriarcado miramos que nuestros derechos como mujeres estaban siendo vulnerados, ya que no teníamos la oportunidad de ejercer la participación organizativa en el territorio y, más aún, en los Consejos Comunitarios. 
Gutiérrez-Rojas y Ramírez-Giraldo

Otra motivación que la llevó a conformar un colectivo de mujeres con más de 350 asociadas fueron los hechos victimizantes que ocurrieron en la región a inicios del año 2000 por parte de las Autodefensas Unidas de Colombia. En el marco del conflicto armado colombiano, un cuñado suyo fue desaparecido y hasta la fecha no se ha sabido nada sobre él. Esta experiencia personal la llevó también a ser activista por el plebiscito por la paz:

Eso fue un choque muy fuerte para nosotros como familia y como víctimas, pero también eso nos conllevó a organizarnos desde la reconciliación y el perdón. Saber que tengo unos familiares dados por desaparecidos me ha dado cada día más motivos para creer en la paz, por la justicia, por la verdad, por la reparación y por la no repetición.

La actividad participativa de este caso de estudio se centró, principalmente, en el colectivo de mujeres que buscaban menguar su dolor, por una parte, y fomentar la participación en consejos comunitarios, por otra. Su participación, como ya se dijo, orbitó sobre la construcción del rol de la mujer afrodescendiente y no sobre la figura del joven como actor político.

Un aspecto a resaltar en este caso es que, a diferencia de los otros tres casos, las políticas públicas sociales, en este caso de juventud, no le han llegado ni la han afectado. En los dos primeros casos de estudio hubo una participación en procesos de formulación de política o en acercamientos con espacios políticos partidistas. En el siguiente caso (institucional) se verá cómo las instituciones creadas por la política forjaron toda una experiencia que muta de lo institucional a lo electoral. Sin embargo, en este caso particularmente, la entrevistada manifestó que nunca fue parte de un proceso de formulación de política o beneficiaria de alguna política estatal, ni por parte de la Alcaldía ni del Departamento.

Aunque la participación electoral e institucional es casi nula, la coocurrencia con la participación alternativa y cívica ha estado presente en el caso. Ha sido partícipe de diferentes protestas y marchas en las que se ha manifestado en contra de la explotación minera en su territorio: "se han hecho marchas porque hace aproximadamente nueve años las concesiones mineras de Canadá y Brasil querían meterse a la fuerza al territorio". 


\section{Caso 4. Institucional}

Figura 5. Trayectoria, caso institucional.

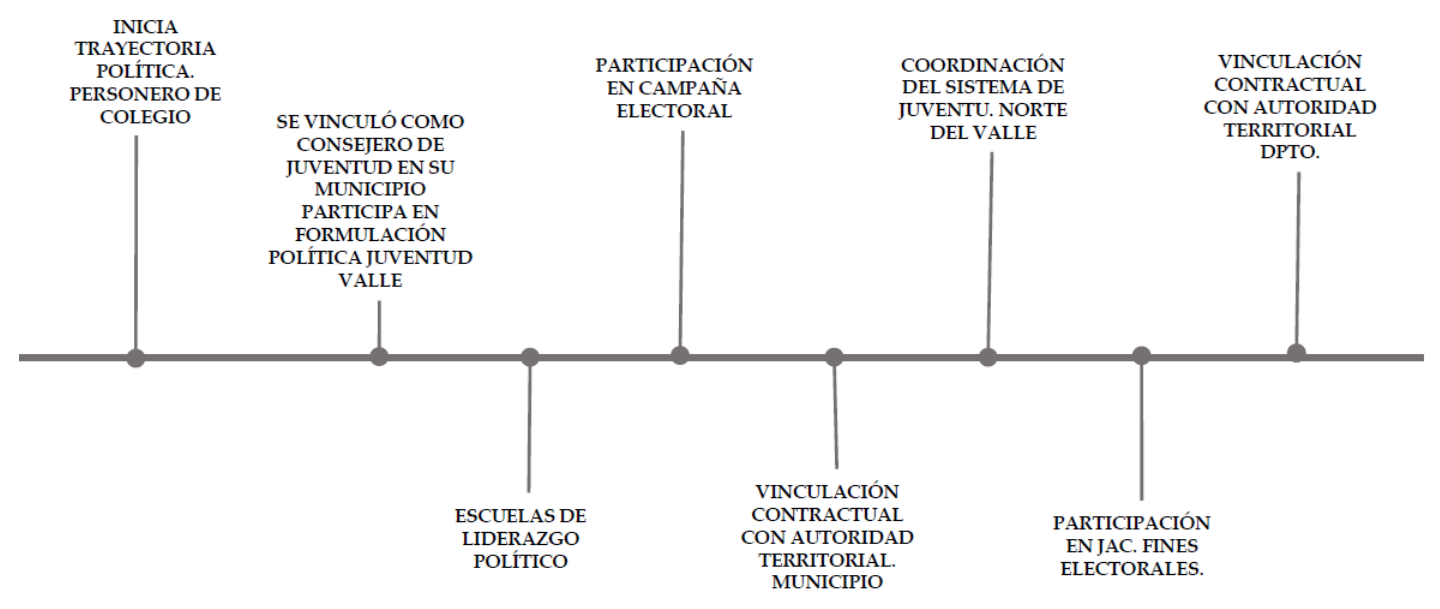

Fuente: elaboración propia.

El último caso de observación es un hombre (Figura 5), quien ha hecho su liderazgo y ha tenido sus experiencias en la subcategoría institucional. Ha sido miembro de los consejos municipales de juventud de su lugar de residencia, participado en plataformas de juventud y asistido a congresos y eventos juveniles, también ha sido miembro y presidente de juntas de acción comunal y ha tenido participaciones en el campo electoral.

Sus primeras experiencias en materia de participación se remontan a la época de colegio, donde fue electo personero. Posteriormente, gracias a iniciativas gubernamentales del Departamento se socializó en su colegio todo lo relacionado con los Consejos Municipales de Juventud creados por la Ley 375 de 1997 y reglamentados por el Decreto 089 del 2000. Estas socializaciones hicieron parte de acciones gubernamentales que fueron puestas en marcha gracias a estos marcos normativos.

Los consejos de juventud permiten o permitieron en ese entonces a los jóvenes que aprovechamos la oportunidad, empezar a abrir brechas y empezar a tener contactos, a tener personas en otros municipios, en otros departamentos, donde se podía aprender, formar y apropiarse de otros escenarios.

Posteriormente, esta experiencia le permitió vincularse a una campaña electoral al apoyar a un candidato a la alcaldía, el cual, una vez obtenido el triunfo, lo nombró miembro del gabinete departamental al vincularlo contractualmente con la administración municipal. Al hacer parte del cuerpo burocrático, siguió ejerciendo actividades relacionadas con temas 
Gutiérrez-Rojas y Ramírez-Giraldo

de juventud y fue nombrado coordinador del sistema departamental de juventudes en 18 municipios del norte del departamento. Nuevamente las coocurrencias se hacen presente en este caso de estudio, pues las diversas formas de participación política están presentes siempre de manera paralela.

Esa participación institucional en conjunto con la electoral lo llevaron también a la participación cívica, donde, siendo aún joven, con 18 años, decidió ser miembro de la junta de acción comunal de su barrio con el fin de alcanzar la presidencia, siempre buscando un fin electoral: la consecución y el mantenimiento de una votación. Aquí se encontró con las primeras barreras actitudinales en su experiencia, pues su jefe político dudó de su capacidad debido a su corta edad. Simultáneamente, fundó un club que realizaba labor social en el municipio de residencia y en los alrededores. Fue también parte de la pastoral social, trabajo comunitario que adelanta la Iglesia católica.

\section{Conclusiones}

Una vez descritos los cuatro casos que se han tomado como referencia para realizar los análisis, hay que añadir un comentario global que dé respuesta a dos de las preguntas de investigación derivadas que se pretendían responder. La primera de estas: ¿Cómo tramitan los jóvenes sus demandas frente al Estado en el nivel territorial (departamento del Valle del Cauca)?, la cual se enlaza directamente con uno de los objetivos específicos que busca describir las experiencias de participación política de los jóvenes en el departamento del Valle del Cauca.

Para dar respuesta a esta interrogante se describieron cuatro casos de dos hombres y dos mujeres que han tenido experiencia en participación política desde diferentes escenarios. Así las cosas, la tramitación de demandas, desde un punto de vista sistémico, se logra a través de cuatro "caminos" que se entrecruzan entre sí: unos lo hacen desde lo electoral, participando en campañas electorales, yendo a las urnas o siendo candidatos a elecciones uninominales o plurinominales; pueden también tomar parte activa en las decisiones participando en los canales institucionales que el Estado dispone para interlocutar con la sociedad civil: plataformas de juventud o consejos de juventud; la otra opción que se ha identificado ha sido la alternativa, la cual consiste en participar a través de la movilización social y ejerciendo presión desde los espacios sociales, colectivos, comunitarios y digitales; por último, está el camino cívico, que implica la participación desde acciones efectuadas que no necesariamente buscan la incidencia política y son contingentes a la toma de decisiones.

Se argumenta que los caminos se cruzan porque los cuatro tipos ideales construidos se interceptan entre sí en todos los sujetos que han sido descritos aquí: la candidata a corporación pública ha participado en procesos de formación políticos alternativos en 
Gutiérrez-Rojas y Ramírez-Giraldo

diferentes universidades públicas; el joven que ha sido personero escolar y consejero de juventud ha sido también partícipe en campañas electorales, lo que le ha permitido vinculación contractual con el Estado; el alternativo que ha forjado su liderazgo político a raíz de un activismo en el tema animalista y medio ambiental, ha estado vinculado a espacios electorales; la joven cívica que ha construido su trayectoria a partir de su identidad de género, ha estado activa en marchas y protestas contra la explotación minera en su territorio. Ninguno de los tipos se presenta en su forma pura, siempre hay yuxtaposiciones que construyen trayectorias que se tornan únicas.

Para dar respuesta a la pregunta de investigación, a continuación, se desglosarán las experiencias por cada tipo ideal construido y analizado previamente en las diferentes categorías aquí planteadas y, en cada uno, se evaluará la incidencia y las relaciones de poder en el marco de la toma de decisiones en política pública.

La categoría electoral presenta una particularidad a nivel departamental, pues tiene una mayor incidencia en las decisiones que el gobierno adopte, ya sea que estén relacionadas con contratación, con priorización de recursos públicos o con la presentación de proyectos y programas; sin embargo, se debe hacer la advertencia que el proselitismo político no garantiza incidir en la agenda pública. Pero se constató que la incidencia de esta joven se da en todas las fases del ciclo de la política, desde el agendamiento, hasta la evaluación por su cercanía con el tomador de decisiones. Las corporaciones públicas son las que deben estudiar y analizar las propuestas que el gobierno presenta para que se aprueben por Acuerdo (municipal) u Ordenanza (departamental). Al ser miembro de una corporación pública, podrá siempre modificar los proyectos que se presenten empleando las herramientas que la ley le brinda. Adicionalmente, en su trayectoria política y al haber estado vinculada a una campaña local y a un partido, pudo llegar a ser asesora personal de un alcalde y decidir sobre la contratación del municipio.

Se argumenta que en este campo de investigación, el electoral, la persona ha tenido en su experiencia un nivel alto de incidencia política en la toma de decisiones al haber estado cercana a los principales ejecutores de las decisiones, ya que se convirtió en la asistente privada del alcalde al haberse vinculado contractualmente con el Estado a nivel territorial.

En lo que respecta al sujeto institucional, sus experiencias de participación han orbitado, principalmente, en los espacios institucionales de que dispone el Estado, como son las Plataformas de Juventud y los Consejos Municipales de Juventud. Su participación en estos espacios le permitió la interlocución y la inclusión de necesidades de los jóvenes en la agenda pública. Gracias al rol desempeñado pudo formar parte del equipo formulador de la política pública departamental de juventud del Valle del Cauca, lo que le permitió incidir en todo el proceso de formulación de manera directa. 
Gutiérrez-Rojas y Ramírez-Giraldo

La capacidad de incidencia política es más baja que en el caso electoral, pues su participación se limita a lo temático juvenil, mientras que la primera categoría, al ser cercana al centro de poder que toma las decisiones, puede incidir en otros aspectos que no son solo los juveniles. Sin embargo, si se analiza la experiencia de participación que ha tenido este mismo sujeto en el campo electoral, su capacidad de incidencia aumenta debido a la vinculación contractual con el Estado y a la cercanía con las autoridades locales y departamentales.

El caso alternativo ha tenido niveles de incidencia similares al caso institucional, puesto que también participó en un proceso de formulación de política juvenil. Esto contaría como participación institucional y no propiamente como alternativa. En esta última categoría la incidencia ha ido de la mano de la movilización social al ser parte de marchas y protestas, y esta se ubica en el agendamiento de situaciones problemáticas que han logrado ejercer presión a las autoridades municipales en temas como las corridas de toros y las cabalgatas.

El sujeto de estudio ha liderado la construcción de una agenda ambiental para el municipio, la cual ha logrado incluir ciertas medidas en los planes de desarrollo que, sin embargo, no han sido implementadas en su totalidad. De acuerdo con lo manifestado por él, la incidencia se ha orientado más hacia la puesta en agenda de temas públicos en materia animalista y ambiental, por tal razón se argumenta que la capacidad de incidencia es baja.

En cuanto a la investigación del caso cívico, la persona ha estado vinculada en procesos en la zona rural de Buenaventura y ha promovido el fortalecimiento de las mujeres para lograr incrementar su influencia en el gobierno local. Sin embargo, no se constatan niveles de incidencia en la toma de decisiones.

Un segundo aspecto que debe tenerse en cuenta es ¿qué tanto han incidido las acciones públicas para el fortalecimiento de los liderazgos? Para el caso electoral, de acuerdo a lo expresado por la entrevistada, se evidencia una influencia directa de políticas públicas como incentivo a la participación, pues las experiencias iniciales se configuran a través de la figura de la personería escolar en el municipio y de la creación de la asociación de personeros municipales. En el caso institucional hay una clara influencia de los espacios de participación para la juventud en la formación de un liderazgo político, pues su trayectoria nace a partir de los procesos de elección en los Consejos Municipales de Juventud y en las Plataformas de Juventud, ambos espacios creados por la normatividad juvenil. Para el caso alternativo hay una influencia marginal con escuelas de liderazgo político que ayudaron a forjar al sujeto, sin embargo, su trayectoria ha estado ligada a la movilización social. Finalmente, en el caso cívico la influencia de las actuaciones del Estado ha sido nula, de acuerdo con lo manifestado por la entrevistada directamente, pues no ha habido socializaciones de las políticas públicas. 
Gutiérrez-Rojas y Ramírez-Giraldo

Como grandes conclusiones hay que anotar, en primer lugar, que la capacidad de incidencia política viene dada por la cercanía al centro de poder en la toma las decisiones. Esto se constata en la trayectoria política del caso electoral y del caso institucional, su capacidad de influir en la toma de decisiones se liga estrechamente no solo con la vinculación contractual, sino con la cercanía política a los decisores. De otro lado, los espacios de diálogo institucional siguen siendo escenarios de interlocución más que puntos de encuentro donde se tomen decisiones; los canales alternativos sirven para movilizar temas e incluir en la agenda lo que interesa a los grupos de presión y la acción cívica no deja de ser tangencial y contingente.

En segundo lugar, los cuatro casos analizados demuestran que los jóvenes tramitan sus demandas de diversas maneras, sin embargo, se percibe un patrón: los casos institucional, alternativo y cívico tienen una trayectoria ligada a un tema, juvenil para el primer caso, animalista para el segundo y de género para el tercero. Esto implica que la incidencia en la toma de decisiones no solo se relaciona con la cercanía a los tomadores de decisión, sino que también va enlazada a una temática particular. El caso electoral, como ya se dijo, presenta unas características diferentes pues su incidencia orbita sobre diversos temas que no son solo temáticos, son generales.

En tercer lugar, en los cuatro casos se perciben también las barreras actitudinales que vienen dadas por lo que en esta investigación se ha denominado adultocentrismo. Para el caso electoral y cívico se le añade una cuestión de género por su condición de mujer, sin embargo, nuevamente la cercanía al centro de poder le sirvió al caso electoral para sortear las dificultades que le trajo ser joven y mujer.

Investigaciones de este tipo son fundamentales para la promoción de la participación política juvenil, pues pensar en la incidencia de los jóvenes en el diseño de políticas públicas en Colombia es entender que el Estado los reconoce como actores sociales y políticos, y no solo como un término que se encuentra en una normatividad.

Finalmente, las políticas públicas y sus intervenciones son herramientas que ejercen una influencia significativa en la formación de liderazgos políticos juveniles. Esto se constata en tres de los casos de estudio (electoral, institucional y alternativo). Estas acciones públicas han rendido frutos cuando la participación política se incentiva en los ciclos de vida tempranos, como la infancia y la adolescencia. 


\section{Referencias bibliográficas}

Acosta-Valencia, G. L., y Garcés-Montoya, Á. (2012). Participación política juvenil. Medellín, Colombia: Sello Editorial Universidad de Medellín.

Almond, G., y Verba, S. (2001). La cultura política. En A. Battle (Ed.), Diez textos básicos de ciencia politica (pp. 171-201). Barcelona: Ariel.

Benedicto, J. (2016). La ciudadanía juvenil: un enfoque basado en las experiencias vitales de los jóvenes. Revista Latinoamericana de Ciencias Sociales, niñez y juventud, 14(2), 925938.

Bobbio, N., y Matteucci, N. (1981). Diccionario de política. México: Siglo Veintiuno.

Botero-Gómez, P., Torres-Hincapié, J., y Alvarado, S. (2008). Perspectivas teóricas para comprender la categoría participación ciudadana - política juvenil en Colombia. Revista Latinoamericana de Ciencias Sociales, niñez y juventud, 6(2), 565-611.

Congreso de Colombia. (4 de julio de 1997). Ley de la juventud [Ley 375 de 1997]. DO: 43.079. Cordourier-Real, C. R. (2015). Participación ciudadana e instituciones: un análisis desde la teoria democrática de Robert Dahl. Estudios sociológicos, 33(99), 579-605. Recuperado de http:// www.scielo.org.mx/scielo.php?pid=S2448-64422015000300579\&script=sci_abstract.

Departamento Nacional de Estadística [DANE]. (2019). Encuesta de Cultura Política (ECP). Información 2019. https://www.dane.gov.co/index.php/estadisticas-portema/cultura/cultura-politica-encuesta.

Díaz-Bravo, L., Torruco-García, U., Martínez-Hernández, M., y Varela-Ruiz, M. (2013). La

Entrevista, Recurso Flexible y Dinámico. Investigación en Educación Médica, 2(7), 162167. http:/ / www.scielo.org.mx/scielo.php?pid=S2007-50572013000300009\&script=sci_arttext.

Díaz-Hernández, P., y Moreno-Martínez, V. (2017). La ausencia del voto por parte de los jóvenes en Colombia (Tesis de maestría). Universidad de Sevilla. Sevilla, España.

Fisher, D. R. (2012). Youth political participation: bridging activism and electoral politics. Annual review of Sociology, 38, 119-137. doi: 10.1146/annurev-soc-071811-145439.

González-Sancho, R., y Henríquez-Cáceres, E. (2016). Participación juvenil en espacios formales de deliberación política: entre adultocentrismo y reproducción del discurso adulto. Revista Rupturas, 7(1), 125-147. doi: 10.22458/rr.v7i1.1614.

Hopenhayn, M. (septiembre, 2004). Participación juvenil y política pública: un modelo para armar. En I Congresso População, pobreza, desigualdade e exclusão na América Latina e Caribe. Associação Latino Americana de População, ALAP, Caxambú, Brasil.

Hurtado-Galeano, D. (2010). Los jóvenes en Medellín: ¿Ciudadanos apáticos? Nómadas (32), 99-115.

Kelly, J. (Coord.) (2003). Políticas públicas en América Latina : teoría y práctica. Caracas, Venezuela: Ediciones IESA.

López-Serrano, J., y López-Serrano, J. (2009). La participación política ciudadana; sus límites y controles institucionales en el caso mexicano. Estudios políticos, 9(16), 9-45. doi: 10.22201/fcpys.24484903e.2009.0.18769. 
Gutiérrez-Rojas y Ramírez-Giraldo

Loreto-Martínez, M., Silva, C., Morandé, M., y Canales, L. (2010). Los jóvenes ciudadanos: reflexiones para una política de formación ciudadana juvenil. Revista Última década, (32), 105-118, Recuperado de https:/ / scielo.conicyt.cl/pdf/udecada/v18n32/art06.pdf.

Müller, P. (2006). Las políticas públicas. Bogotá: Universidad Externado de Colombia.

Niño-Lim, N. (2009). Novel or Novice: exploring the contextual realities of youth political participation in age of social media. Philippine Sociological Review, 57, 61-78. Recuperado de http://www.jstor.org/stable/23898344.

Parker-Gumucio, C. (2003). Abstencionismo, juventud y política en Chile actual. Revista de Estudios Avanzados Interactivos, 4(23), 1-23. Recuperado de https:/ /27d7b3bc-fba4-441bbde1-eacc93750356.filesusr.com/ugd/c6b930_ed94648bd4214e79af0a4f84e9b553f1.pdf.

Pasquino, G. (2011). Nuevo curso de ciencia política. México: Fondo de Cultura Económica.

Presidencia de la República de Colombia. (7 de febrero de 2000). [Decreto 089 de 2000]. DO: 43882.

Roth-Deubel, N. (2017). Políticas públicas: formulación, implementación y evaluación. Bogotá: Ediciones Auroras.

Strauss, A., y Corbin, J. (2002). Bases de la investigación cualitativa: técnicas y procedimientos para desarrollar la teoría fundamentada. Colombia: Editorial Universidad de Antioquia. Facultad de Enfermería de la Universidad de Antioquia. Recuperado de http:/ / www.academia.edu/download/38537364/Teoria_Fundamentada.pdf.

Yin, R. (2017). Case study research and applications: design and methods. Los Angeles: SAGE. 


\section{OTROS ARTÍCULOS DE PROSPECTIVA No. 32 DE 2021}

\section{EDITORIAL}

Compromisos, dilemas y desafíos del Trabajo Social con dimensión colectiva en tiempos de pandemia

Enrique Pastor-Seller

\section{ARTÍCULOS}

La narrativa biográfica como alternativa para la comprensión del Conflicto y la violencia en Colombia: una experiencia pedagógica universitaria

Sara Sofía Castaño-Barco y Claudia BermúdezPeña

Experiencias de transición a la vida adulta de jóvenes que migraron solos. Un estudio en Aragón (España) Daniel Jiménez-Franco; Raquel Berzosa-Callén y Chabier Gimeno-Monterde

Fundamentals of Education in Intervention with Young Offenders. A Critical Review of a 'Successful' Case in Andalucía, España

David Herrera-Pastor

Subjetividad juvenil: lecturas desde y para el Trabajo Social en Colombia

Daniela Joya-Valbuena

Participación politica de los jóvenes del Valle del Cauca, Colombia, en la toma de decisiones públicas

Luis Eduardo Gutiérrez-Rojas y Amanda Ramírez-Giraldo

Victimización y perpetración de violencia en pareja adolescente y redes de apoyo en Colombia. Análisis con perspectiva de género

Johanna Alexandra Reina-Barreto
Apoyo social percibido, autoestima y maternidad adolescente: entre el respeto y la intrusión. Estudio en Traiguén, Chile

Gloria Mora-Guerrero, Luisa Escárate-Colín, Carol Espinoza-Lerdón y Andrea Peña-Paredes

El desarrollo en el Trabajo Social de Colombia: un campo transversal, disperso y polivalente

Maira Judith Contreras-Santos

Análisis de la situación laboral de las alimentadoras en la caficultura de la zona central colombiana, a la luz de la teoría de Nancy Fraser

Pablo Andrés Arango-Giraldo

Prioridades de investigación en la Obra social de empleados públicos, Mendoza, Argentina. Un estudio Delphi sobre producción de conocimientos en la seguridad social

Cecilia Amalia Molina

Producción de conocimiento y toma de decisiones. Relaciones entre academia y política pública para las familias en Colombia

José Raúl-Ruíz y Sandro Leonardo MunévarVargas

El staff de prácticas profesionales en Trabajo Social: espacio de fomento del aprendizaje autodirigido. Un estudio de caso en la Universidad Católica Luis Amigó, Colombia

Cristian Eduardo Blanco-García

\section{ARTISTA INVITADO}

Margie Reinel-Aguilar Ao Cicadidae

Prospectiva

\section{PROSPECTIVA Revista de Trabajo Social e Intervención Social}

No. 32 • jul.-dic. 2021

e-ISSN: 2389-993X • Universidad del Valle 